\title{
I. A simple explanation of Talbot's bands
}

\section{Arthur Schuster F.R.S.}

To cite this article: Arthur Schuster F.R.S. (1904) I. A simple explanation of Talbot's bands, Philosophical Magazine Series 6, 7:37, 1-8, DOI: 10.1080/14786440409463082

To link to this article: http://dx.doi.org/10.1080/14786440409463082

册 Published online: 08 Jun 2010.

Submit your article to this journal $\pi$

Џll Article views: 5

Q View related articles $\asymp$

7 Citing articles: 3 View citing articles 준 
THE

LONDON, EDINBURGH, AND DUBLIN

PIIILOSOPHICAL MAGAZINE

AND

JOURNAT, OF SCIENCE.

[SIXTH SERIES.]

$J A N U A R Y 1904$.

I. A Simple Explanation of Talbot's Bands. By Arthur Sohuster, F.R.S.*

1. THESE bands are observed in a spectrum when half the aperture of the pupil is covered with a thin plate of mica or glass, provided that the plate be inserted on that side on which the blue of the spectrum appears. The explanation of these bands which has been given by Airy and Stokes involves a rather elaborate mathematical process which, though convincing, does not leave the mind completely satistied. The essential reason for the want of symmetry which canses the bands to appear only when the plate is introduced on one side, ought to be capable of being rendered obvious in a more simple manner. This I propose to do in the present communication.

As the bands are seen with "white light," a single luminous impulse should be sufficient to produce them, and as the distribution of intensity in the spectrum is clearly not an essential factor in the ease, we may choose the shape and duration of the impulse as we like.

Let an indefinitely short impulse spread out from a distant point, and strike a plane grating $G G^{\prime}$ normally. This grating may be imagined to be made up of a series of narrow reflecting parallel strips $A_{1}, A_{2}$, \&c., separated by intervals which reflect no light. $A$ lens $S^{\prime} S^{\prime}$ having its focus at $F$ receives the luminous disturbance. The impulsive velocity spreading from $A_{1}$ reaches $F$ sooner than that reflected from

* Communicated by the Author.

Phil. Mag. S. 6. Vol. 7. No. 37. Jan. 1904. 
$\mathbf{A}_{2}$, and so on as the optical distances of the reflecting strips gradually increase from $A_{1}$ to $A_{n}$. Hence the disturbance at F consists of a series of impulses, following each other at intervals equal to the period of a homogeneous wave which, starting from the same luminous point, and reflected by the grating, would have its first principal maximum at $\mathbf{F}$. All this, of course, is simply the elementary illustration, first given, I believe, by Lord Rayleigh, of the ordinary action of a grating when analysing white light. The question now is : How can the impulses which succeed each other at F be made to interfere? Clearly only by retarding those which first reach $\mathrm{F}$, or accelerating those which reach that point last. A plate of appropriate thickness introduced from the left-hand side of the figure as it is drawn, can be made to answer

Fig. 1.

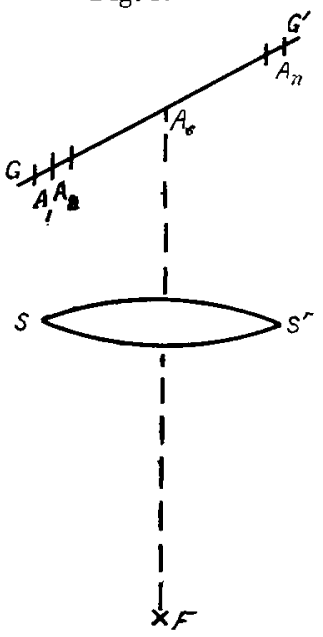
the purpose. If, on the contrary, the same plate were introduced on the right-hand side, it would only retard those impuises which already arrive late, and therefore no interference could take place. This is really all that need be said in explanation of the bands ; but a more detailed consideration of this view of the problem leads easily to a clearer expression for the calculation of the best thickness of the interposed plate than the more elaborate calculations of previous investigations.

The best thickness is secured when the whole series of impulses is divided into two equal portions, the impulses arriving in pairs simultaneously at $F$. If $A_{s}$ be the central line of the grating, the retardation ought to be such that the impulses coming from $A_{1}$ and from $A_{s}$ reach $F$ at the same time. If $\mathrm{N}$ be the total number of lines of the grating, the best retardation is therefore $\frac{1}{2} N \lambda$, and the plate should be pushed sufficiently far into the beam to affect half its width. The wave-length $\lambda$ here means the wave-length of that homogeneous train of waves which has its first principal maximum at $F$, so that the retardation of each impulse compared with the next is $\lambda$. If the retardation is either greater or smaller, some of the impulses arrive too soon or too late to overlap others, and the bands are less clear. If the retardation has more than twice its best value, the series of impulses from $A_{1}$ to 
$A_{s}$ pass through $\mathbf{F}$ later than those coming from $\mathbf{A}_{\boldsymbol{s}}, \mathbf{A}_{\boldsymbol{s}+1}$, \&c., and hence there cannot be any interference.

If at a certain point of the spectrum corresponding to a wave-length $\lambda$ there is a maximum of light, the relative retardation of the two interfering impulses must be equal to $m \lambda, m$ being an integer; the next adjoining band towards the violet will appear at a wave-length $\lambda^{\prime}$ such that

$$
m \lambda=(m+1) \lambda^{\prime} .
$$

Hence for the distance between the bands

$$
\frac{\left(\lambda-\lambda^{\prime}\right)}{\lambda^{\prime}}=\frac{1}{m},
$$

with the best thickness of interposed plate, $m=\frac{1}{2} \mathrm{~N}$, and hence

$$
\frac{\lambda-\lambda^{\prime}}{\lambda^{\prime}}=\frac{2}{\mathrm{~N}^{*}}
$$

where $\lambda^{\prime}$ in the denominator may with sufficient accuracy be replaced by $\lambda$. If $\lambda^{\prime \prime}$ be that wave-length nearest to $\lambda$ at which there is a minimum of light, it follows that

$$
\frac{\lambda-\lambda^{\prime \prime}}{\lambda}=\frac{1}{\mathrm{~N}} \text {. }
$$

If a linear homogeneous source of light of wave-length $\lambda$ be examined by means of a grating, the central image extends to a wave-length $\lambda_{1}$ such that

$$
\frac{\lambda-\lambda_{1}}{\lambda}=\frac{1}{N},
$$

where $\mathrm{N}$, as before, is the total number of lines on the grating. Hence the following proposition :- -If, in observing Talbot's bands, that thickness of retarding-plate be chosen which reduces the minimum illumination of the dark spaces to zero, the distance between each maximum and the nearest minimum is equal to the distance between the central maximum and the first minimum of the diffractive image of homogeneous light, observed in the same region of the spectrum with the same optical arrangement. This proposition holds for all orders of spectra; bat the appropriate thickness of the retarding plate increases in the same proportion as the order.

Lord Rayleigh's remark * that "the thickness of the plate must not exceed a certain limit, however pure the spectrum may be," requires the qualification that for infinite purity the limiting thickness also becomes infinite.

To examine the case in which the thickness of the retarding * 'Encyclopædia Britannica' and 'Collected Works,' vol, iii. p. 133. 
plate is not that which gives no light at the minimum, consider a certain retardation $A_{1} B_{1}$ introduced into half the beam. The effect is the same as if the impulses, instead of starting from $\mathrm{A}_{1}, \mathrm{~A}_{2}, \ldots \mathrm{A}_{s}$, were sent off simultaneously from $\mathrm{B}_{1}, \mathrm{~B}_{2}, \ldots \mathrm{B}_{8}$, where $\mathrm{A}_{1} \mathrm{~B}_{1}=\mathrm{A}_{2} \mathrm{~B}_{2}=\ldots \mathrm{A}_{8} \mathrm{~B}$ ( (fig. 2). If $\mathrm{B}_{1} \mathrm{~A}_{p}$ and $\mathrm{B}_{8} \mathrm{~A}_{g}$ are lines drawn parallel to the principal plane of the focussing-lens so that the optical distances of $B_{1}$ and $A_{p}$ and of $B_{s}$ and $A_{q}$ to the focus are the same, the impulses starting from points between $\mathrm{B}_{f}$ and $\mathrm{B}_{s}$ may interfere with those starting from points between $\mathrm{A}_{s}$ and $\mathrm{A}_{g}$; but Fig. 2.

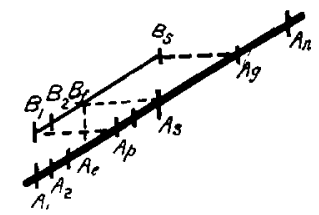
the parts of the grating $A_{1} A_{e}$ and $A_{g} A_{n}$ are not effective as regards interference, the impulses from $A_{1} A_{e}$ arriving too soon and those from $A_{g} A_{n}$ too late.

If the useless portions are cut off by means of screens, the bands become black again, and we return to the best retardation, but with a reduced aperture, and consequently a reduced resolving power. The introduction of these screens does not alter the width of the bands; but these will be further apart than for the best retardation on account of the reduced cross-section of the effective portion of the beam. If the retarding plate is thicker than that which gives the most distinct bands, it may similarly be shown that the blackness of the darkest portions may be restored by screening off the central portions of the beam; but the bands will be closer together in this case. The point at which the bands disappear altogether is that at which each homogeneous component of the impulse gives a central diffraction-image equal in width to the distance between the bands. In each case the width of the bands is easily seen to be the same as that given by the interference of two points of light separated laterally in the beam by a distance equal to that of any two of the pulses which reach the focus simultaneously.

Powell's experiment in which bands are obtained by introducing plates into part of the beam traversing a hollow prism filled with a refracting substance is only a modification of Talbot's, and is explained in a similar manner.

2. An objection might be raised to the above explanation in so far as it is not perhaps at once obvious how darkness may result by the interference of a succession of impulses which are all in the same direction. The answer to the objection is, that if the impulses belonging to the first half of the beam are retarded in such a way as to fit in exactly half-way between those belonging to the second half, the 
disturbance at $\mathrm{F}$ would be a succession of impulses corresponding to the half period of the original impulse. There is light at $\mathrm{F}$, but it is light which belongs to the overlapping spectrum of the second order. As regards the wave-length $\lambda$ under consideration, there is darkness. The difficulty, if it is still felt to be one, may be avoided by considering a grating giving rise to the "corrugated waves" of Lord Rayleigh*. I have called these gratings " simple gratings," as all other gratings may be imagined to be made up of superposed simple gratings. The light of a simple grating is concentrated into the two spectra of the first ordert. It may readily be shown that any device which gets rid of the spectra of different orders will change impulses which were originally in one direction into disturbances which are alternately in one and the other direction, so that no further question can arise as to the way in which, according to the view here adopted, the dark bands are formed in Talbot's experiment.

3. The proposition proved in $\S 1$ allows us to extend the investigation to the case where the spectrum is produced by a prism. In the immediate neighbourhood of a given wavelength, the spectrum may be taken to be a normal one, and there can be no intrinsic difference between the bands seen in this case and those observed when a grating of the same resolving power is used. It has been shown by Lord Rayleigh that in all questions relating to resolving power the number of lines in the grating has to be replaced in the case of prisms by $t \frac{d \mu}{d \lambda}$, where $t$ is the aggregate effective thickness of the prisms, $\mu$ the refractive index, and $\lambda$ the wave-length. It follows at once that the retardation which gives black bands is for prisms

$$
\frac{1}{2} \lambda t \frac{d \mu}{d \lambda} .
$$

4. It is interesting to follow out the modus operandi of a prism when an impulse is transmitted through it. For the sake of simplicity we may confine ourselves to the case that the law of refraction is such that the group velocity is independent of the wave-length. If the impulse be confined to the wave-front W F (fig. 3 ) before entering a refracting substance, it will at a given time in its passage through it

* 'Encycloprdia Britannica,' "Wave Theory," and 'Collected Works.'

† Phil, Mag. xxxvii. p. 509 (1894). 
lie near some surface $G G^{\prime}$ which moves forward with the group velocity. I have shown, in a paper communicated to the recent meeting of the British Association, that the shape of the impulse changes periodically and alternately passes through its original shape and one exactly equal but opposite in direction. If now the impulse has passed through the prism and a wave-front for a homogeneous wave of length $\lambda$ would lie in the direction RS, the "impulses" will be confined to a region immediately surrounding a plane $\mathrm{HS}$, the position of which may be calculated by the ordinary law of refraction, substituting the group velocity for the wave velocity. But on $\mathrm{HS}$ the impulsive motion is not uniform, but alters periodically from the original type to that which is equal and opposite to it. Hence if the emergent beam be received

Fig. 3.
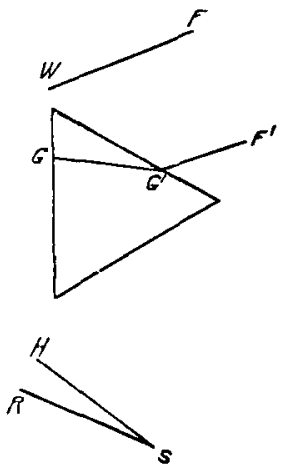
by a lens, the disturbance at the focus of the lens consists of a periodic motion which is the more homogeneous the greater the resolving power of the prism. It will be noticed that this explanation of the modus operandi of a prism differs materially from that given by Dr. J. Larmor ('Ather and Matter,' p. 248); but as we may imagine continuous media of such elastic properties as to give dispersion, the true explanation must be independent of the sympathetic vibrations which Dr. Larmor calls to his aid. To calculate the angle between $\mathrm{RS}$ and $\mathrm{H} \mathrm{S}$, we note that $H \mathrm{R}$ is equal to the space passed over in air in the time equal to the difference between that necessary to traverse the thickness $t$ of the prism when the veloeity is that of a homogeneous wave and when it is that of the group. Hence $\mathrm{U}$ being the group velocity, $\mathrm{V}$ the wave velocity in vacuo, and $V^{\prime}$ the wave velocity in the prism,

$$
\begin{aligned}
\mathrm{RH} & =\mathrm{V}\left(\frac{t}{\mathrm{U}^{\prime}}-\frac{t}{\mathrm{~V}^{\prime}}\right) \\
& =\frac{\mathrm{V} t}{\mathrm{UV}^{\prime}}\left(\mathrm{V}^{\prime}-\mathrm{U}\right),
\end{aligned}
$$

and if $\mu=\mathrm{V} / \mathrm{V}^{\prime}$ be the refractive index,

$$
=\frac{\mu t}{\mathrm{U}}\left(\mathrm{V}^{\prime}-\mathrm{U}\right) \text {. }
$$


But $k$ being the frequency,

hence

$$
\begin{aligned}
\mathrm{U} & =\frac{d k \mathrm{~V}^{\prime}}{d k} \\
& =\mathrm{V}^{\prime}+k \frac{d \mathrm{~V}^{\prime}}{d k} \\
& =\mathrm{V}^{\prime}-\lambda \frac{d \mathrm{~V}^{\prime}}{d \lambda}=\mathrm{V}^{\prime}+\frac{\lambda}{\mu} \mathrm{V}^{\prime} \frac{d \mu}{d \lambda} .
\end{aligned}
$$

$$
\mathrm{RH}=\stackrel{\lambda V^{\prime} t}{\mathrm{U}^{-}} \frac{d \mu}{d \lambda} .
$$

We may put with sufficient accuracy $\mathrm{V}^{\prime}=\mathrm{U}$ in this expression. To observe Talbot's bands, the retarding plate must be brought in on the side of the thin edge of the prism and the best thickness, according to $\S 1$, is that in which that half of the beam which is nearest the thin end of the prism is retarded through half the distance $\mathrm{RH}$. The appropriate thickness is therefore $\frac{\lambda t}{2} \frac{d \mu}{d \lambda}$ in accordance with the results of the previous paragraph.

5 . The previous investigation gives the retardation which the plate should produce if the best effect is to be observed. If we wish to determine in an actual case the best thickness of plate, wa must remember that as we have been dealing with impulses the group velocity comes into play. Hence the usual expression $(\mu-1) e$ for the retardation, the thickness being $e$, is not quite accurate.

If $U$ be the group velocity, and $V^{\prime}$ the velocity of light in vacuo, the retardation in time is $e\left[\frac{1}{U}-\frac{1}{V^{\prime}}\right]$; this corresponds
to a distance in air of

$$
e[\overline{\mathrm{U}}-1] \text {, }
$$

or if $\mathrm{V}$ be the velocity in the substance of the plate, the retardation is

$$
e\left[\mu^{\prime} \frac{\mathrm{V}}{\mathrm{U}}-1\right],
$$

$u^{\prime}$ being the refractive index of the material of the retarding plate.

We obtain the right result by adding to $(\mu-1) e$ the distance through which the group has fallen behind the wave; this corresponds to the quantity $\mathrm{RH}$ calculated as above, if for the thickness of the prism we substitute $e$ and write $\mu^{\prime}$ 
for the refractive index of the plate. This gives for the retardation

$$
e\left(\mu^{\prime}-1-\lambda \frac{d \mu^{\prime}}{d \lambda}\right)
$$

and for the best thickness of the plate this must be equal to $\frac{1}{2} \mathrm{~N} \lambda$ or $-\frac{1}{2} \lambda t \frac{d \mu}{d \lambda}$ according as a grating or prism is used to produce the spectrum.

6. We are so much accustomed to regard the homogeneous wave as the simplest element into which all wave-motions may be resolved that we sometimes overlook the fact that the phenomena of white light may all be reproduced by a single disturbance of short duration. There are cases, and the phenomenon of Talbot's bands may serve as a conspicuous example, where the consideration of the combined group yields to a simpler treatment than the resolution into homogeneous waves. I have shown in my paper on "Interference Phenomena" how group velocities may he used to determine the conditions of achromatism. Considerations similar to those used in that paper may perhaps be usefully employed to simplify the treatment of achromatized interference-bands.

II. On the Variation of Entropy as treated by Prof. Willard Gibbs. By H. A. Bumstead, Ph.D., Assistant Professor of Physics, Yale University *.

TN the August number of the Philosophical Magazine Mr. S. H. Burbury bas discussed certain difficulties which present themselves in Chapter XII. of the "Principles of Statistical Mechanics," by the late Prof. J. Willard Gibbs. Unless I have misunderstood Mr. Burbury's statement, I believe these difficulties may be surmounted, and shall endeavour to give, as briefly as possible, my reasons for this belief. For the sake of brevity I shall assume that the reader has Mr. Burbury's paper before him, and shall refrain from quoting from it unless it seems necessary for clearness of statement.

The first difficulty (which may be more conveniently discussed in terms of the hydrodynamical analogue than in terms of the ensemble of systems) is in regard to Prof. Gibbs's statement that "one may perhaps be allowed to say that a finite amount of stirring will not affect the meau square of the density of the colouring matter, but an infinite amount of stirring may be regarded as producing a condition

* Communicated by the Author. 